\title{
Stiffness Estimation and Nonlinear Control of Robots with Variable Stiffness Actuation
}

\author{
Fabrizio Flacco Alessandro De Luca \\ Dipartimento di Informatica e Sistemistica \\ Università di Roma "La Sapienza", Via Ariosto 25, 00185 Roma, Italy \\ \{fflacco,deluca\}@dis.uniroma1.it
}

\begin{abstract}
We consider the problem of estimating on line the nonlinear stiffness of flexible transmissions in robots with variable stiffness actuation in agonistic-antagonistic configuration. Stiffness estimation is obtained using a dynamic residual that provides a filtered version of the unmeasured flexibility torques, combining it with a recursive least squares algorithm that fits a polynomial model to the data, and proceeding then by analytical derivation. Only motor position/velocity and link position measurements are used, while knowledge of dynamic parameters is required for the motors but not for the links. The estimated stiffness function, together with its first two derivatives with respect to the deformation, is used within a feedback linearization controller designed for simultaneous tracking of desired trajectories for the links and the device stiffnesses. Simulation results provided for the VSA-II device demonstrate the perfomance of the estimation process and the effectiveness of the complete control approach.
\end{abstract}

Keywords: Flexible transmissions, Variable stiffness actuation, Stiffness estimation, Recursive least squares, Feedback linearization control, Robot motion control

\section{INTRODUCTION}

For a safer physical Human-Robot Interaction (pHRI), compliant elements are introduced in robot manipulators at different levels in the structure, including end-effector tools, link surfaces, and robot joints, transmissions, and actuation devices (De Santis et al. (2008)). A compliant (non-stiff) robot allows to milden the danger of injuries to the human user due to accidental collisions. In particular, the use of flexible transmissions reduces the effective inertia seen during a dynamic impact with the environment thanks to the mechanical decoupling between the relatively large motor inertias and the inertia of the (lightweight) robot links. On the other hand, flexible transmissions will challenge the control performance in preventing vibrations, accurately tracking reference trajectories, and limiting energy consumption (De Luca and Book (2008)).

One recent trend in pHRI is to design robots using variable stiffness actuation (VSA) devices, see Bicchi and Tonietti (2004); Tonietti et al. (2005); Wolf and Hirzinger (2008); Catalano et al. (2010). In this context, the mechanical stiffness of a robot joint is defined as the relation between the displacement at the link side of the transmission and the flexibility torque that arises in reaction. The joints of an industrial robot equipped with harmonic drives display a constant stiffness, i.e., the transmissions can be modeled by springs that work in their linear elastic domain. In contrast, in order to obtain a (passive or active) variation of joint stiffness during robot motion, the flexible transmission should behave in a nonlinear way. This can be obtained either through a spring with nonlinear (e.g., cubic or exponential) deformation-torque characteristic or with an elastic spring of constant stiffness mounted in a nonlinear kinematic arrangement. In VSA-based robots, two independent motors are used at each joint and motion is transmitted through nonlinear flexible transmissions assembled in various configurations. One example, which will be used in this paper, is given by the VSA-II device developed at the University of Pisa by Schiavi et al. (2008), where the two actuators work in a parallel, agonisticantagonistic, bi-directional, and (nominally) symmetric mode. With two actuators, it is possible to actively control the motion of the links/load while modifying on line (from softer to harder and viceversa) the stiffness of the joints. A safe and energy efficient behavior is obtained, e.g., by imposing a small stiffness at high link velocities and a large stiffness at low velocities, as in the safe brachistochrone planning solution introduced by Bicchi and Tonietti (2004).

Feedback control laws intended for regulation and/or trajectory tracking in robots with flexible joints typically need a good knowledge of the robot dynamic parameters, including joint stiffness, see, e.g., De Luca and Book (2008); Tonietti et al. (2005). Under the ideal assumptions that a perfect model is available and that the full robot state is measured, De Luca et al. (2009) have shown that a single link moving under gravity and driven by a VSA antagonistic device can be exactly linearized by means of nonlinear feedback. This result can be easily extended to the general multi-link case. The proposed feedback linearization design enables simultaneous and decoupled control of link motion and joint stiffness, achieving exponentially stable tracking of sufficiently smooth reference profiles for these quantities.

Unfortunately, there are no available sensors for a direct measure of stiffness. This physical quantity is usually 
computed from position and/or joint torque sensor data, based on a nominal model and static calibration. This procedure is especially critical in VSA-based robots, since the stiffness is intrinsically a nonlinear function of the joint deformation, its model may have a quite complex (and uncertain) expression, and the rigid body dynamics of the driven robot links is highly nonlinear. In addition, since the device stiffness that should be controlled is not directly measured, an intrinsic robustness limitation arises: at best, the nominal or estimated stiffness output (and not the actual one) can track the desired reference.

The above considerations motivated the need for online methods of stiffness estimation in VSA-based robots. While some authors have dealt with stiffness estimation in the contact between the end-effector of a rigid robot and the environment/human (see, e.g., Diolaiti et al. (2005); Verscheure et al. (2009); Ludvig and Kearney (2009); Coutinho and Cortesao (2010)), work on estimation of variable, nonlinear stiffness of single- or double-actuated flexible joints is still at the beginning. Grioli and Bicchi (2010) have introduced a stiffness estimator based on the knowledge of the flexibility torque, which is in turn explicitly measured by a sensor. Their estimator processes the time derivative of the measured flexibility torque, which may give problems due to noise. A batch (almost on-line) estimation method that does not use joint torque sensing nor acceleration estimation has been recently proposed by Flacco and De Luca (2011).

In this paper, we present a new method for on-line stiffness estimation for VSA-based robots and its use within a feedback linearization control scheme. The developments are made for a single-dof agonistic-antagonistic system under gravity, but can be easily extended to multi-dof robots. For stiffness estimation, we will only use the knowledge of dynamic parameters (inertia and viscous friction coefficient) of the two motors and measures of the motor position and velocity and of the link position. Our estimation approach uses two basic tools: $i$ ) a residual-based method, as inherited from FDI techniques (see, e.g., De Luca and Mattone (2003); Haddadin et al. (2008)), which provides a filtered version of the (unmeasured) flexibility torques at the joint, and ii) a standard Recursive Least Squares (RLS) algorithm that fits the residual data to a general parametrized model, which is chosen as a polynomial in the transmission deformation. From this estimated model, we can compute analytically the stiffness and its first two derivatives with respect to the joint deformation, as needed by the feedback linearization controller of De Luca et al. (2009). Based on the certainty equivalence principle, the resulting on-line estimates are fed into the nonlinear controller. The proposed estimation/control approach is summarized in the scheme of Fig. 1.

The paper is organized as follows. In Sect. 2 we present the dynamic modeling framework. The residual design for online estimation of the flexibility torque is given in Sect. 3 . The model-based estimation of transmission stiffness using a RLS algorithm is discussed in Sect. 4, together with an error recovery scheme to compensate for the filtering action of the residual (Sect. 4.1). In Sect. 5, numerical results on stiffness estimation are reported for the VSA-II device moving a link under gravity. Finally, Section 6 revisits the feedback linearization control law introduced

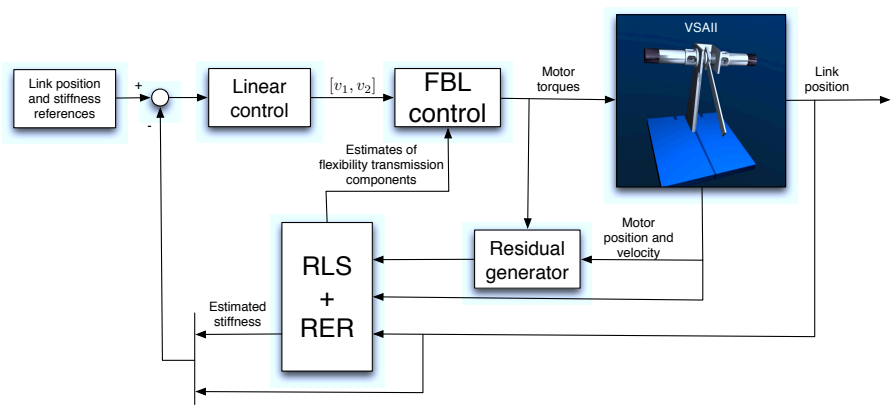

Fig. 1. Scheme of stiffness estimation and its use in feedback linearization control

by De Luca et al. (2009) when on-line estimates of all quantities related to stiffness are used, and illustrates the obtained closed-loop performance by simulations.

\section{DYNAMIC MODELING}

Consider a flexible transmission that connects a driving motor to the driven link. The deformation $\phi=q-\theta$ of the transmission is the difference between the motor angle $\theta$ and the link angle $q$. A potential function $U_{e}(\phi) \geq 0$ is associated to the deformation $\phi$, with $U_{e}(\phi)=0$ iff $\phi=0$. The flexibility torque across the transmission is $\tau_{e}(\phi)=\partial U_{e}(\phi) / \partial \phi$, and based on physical arguments and current implementations it can be assumed that

$$
\tau_{e}(0)=0, \quad \tau_{e}(-\phi)=-\tau_{e}(\phi), \quad \forall \phi .
$$

The stiffness of the transmission is defined as the variation of the flexibility torque w.r.t. link displacements

$$
\sigma(\phi)=\frac{\partial \tau_{e}(\phi)}{\partial q}=\frac{\partial \tau_{e}(\phi)}{\partial \phi}>0
$$

For a single motor driving a rigid link subject to gravity through a (nonlinear) flexible transmission, the dynamic model takes the form

$$
\begin{aligned}
M \ddot{q}+D_{q} \dot{q}+\tau_{e}(\phi)+g(q) & =0 \\
B \ddot{\theta}+D_{\theta} \dot{\theta}-\tau_{e}(\phi) & =\tau,
\end{aligned}
$$

where $M>0$ and $B>0$ are the link and motor inertias, $D_{q} \geq 0$ and $D_{\theta} \geq 0$ are the viscous friction coefficients at the two sides of the transmission, $\tau$ is the torque provided by the motor (after gear reduction), and $g(q)$ is the gravity torque acting on the link.

For an agonistic-antagonistic variable stiffness actuation device, e.g., those in Bicchi and Tonietti (2004); Schiavi et al. (2008), two motors are connected in parallel to the link through two (possibly, different) nonlinear flexible transmissions (see Fig. 2). With obvious notation, the model becomes

$$
\begin{aligned}
M \ddot{q}+D_{q} \dot{q}+\tau_{e, t}(\phi)+g(q) & =0 \\
B_{i} \ddot{\theta}_{i}+D_{\theta, i} \dot{\theta}_{i}-\tau_{e, i}\left(\phi_{i}\right) & =\tau_{i}, \quad i=1,2 .
\end{aligned}
$$

Accordingly, the stiffnesses of the two transmissions are

$$
\sigma_{i}\left(\phi_{i}\right)=\frac{\partial \tau_{e, i}\left(\phi_{i}\right)}{\partial q}=\frac{\partial \tau_{e, i}\left(\phi_{i}\right)}{\partial \phi_{i}}>0, \quad i=1,2
$$

The total flexibility torque $\tau_{e, t}$ in the link dynamics (5) is a separable function of vector $\boldsymbol{\phi}=\left(\phi_{1} \phi_{2}\right)^{T}$ of transmission deformations $\phi_{i}=q-\theta_{i}, i=1,2$, namely 


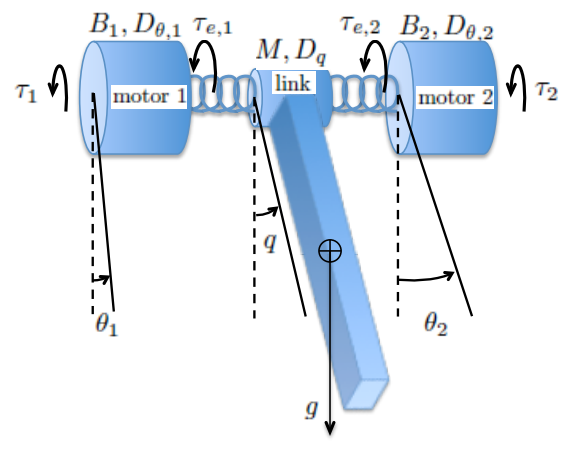

Fig. 2. Definition of variables in a VSA with agonisticantagonistic configuration, with two motors used to drive a single link and modify the device stiffness

$$
\tau_{e, t}(\phi)=\tau_{e, 1}\left(\phi_{1}\right)+\tau_{e, 2}\left(\phi_{2}\right)
$$

The total device stiffness is thus

$$
\sigma_{t}(\phi)=\frac{\partial \tau_{e, t}(\phi)}{\partial q}=\sigma_{1}\left(\phi_{1}\right)+\sigma_{2}\left(\phi_{2}\right)>0 .
$$

If the device is symmetric, one has the same functional form $\tau_{e, 1}=\tau_{e, 2}$ (and thus, $\sigma_{1}=\sigma_{2}$ ), although these functions are still evaluated with dynamically different arguments (namely, $\phi_{1}(t) \not \equiv \phi_{2}(t)$ ).

\section{RESIDUAL APPROACH FOR FLEXIBILITY TORQUE ESTIMATION}

Our goal is to estimate the stiffness $\sigma_{i}$ of each transmission, and thus the total stiffness $\sigma_{t}$ of the VSA device through (9). This can be accomplished without the need of additional sensors other than the encoders available at the motor and link sides for measuring position. While motor velocities will be obtained by numerical differentiation/filtering of position measures, we will not resort to acceleration measurements (or to further derivation of data) nor to torque sensing.

As a preliminary step ${ }^{1}$, the estimation of the flexibility torque $\tau_{e}$ associated to a transmission or to the full device is considered. We follow a residual-based design, which allows in general to generate filtered versions of unknown or uncertain dynamic terms. This tool, in conjunction with the concept of generalized momentum, has been used already for detecting and isolating actuator faults in multidof robot arms (De Luca and Mattone (2003)).

For the problem at hand, we use the generalized momentum of the motor $p_{\theta}=B \dot{\theta}$ and define the following residual:

$$
r_{\tau_{e}}=K_{\tau_{e}}\left(p_{\theta}+D_{\theta} \theta-\int_{0}^{t}\left(\tau+r_{\tau_{e}}\right) d t_{1}\right),
$$

where $K_{\tau_{e}}>0$ is a design parameter and $r_{\tau_{e}}(0)=0$ for a system initially at rest. The evaluation of $r_{\tau_{e}}$ involves only the motor variables $\theta$ and $\dot{\theta}$, as well as the applied input torque $\tau$. Note that no assumption is made on the structure of $\tau$, which can be either an open-loop command or the torque provided by a linear or nonlinear feedback law (in particular, one that uses the estimate itself).

\footnotetext{
1 For notational simplicity, in the following we will often omit the subscripts $i$ or $t$ being these clear from the context.
}

With reference to eq. (6), it is easy to check that the residual $r_{\tau_{e}}$ satisfies

$$
\dot{r}_{\tau_{e}}=K_{\tau_{e}}\left(\tau_{e}-r_{\tau_{e}}\right),
$$

resulting in a stable, first-order filter of the unknown flexibility torque $\tau_{e}$ (i.e., of $\tau_{e, i}$ for the $i$ th transmission). In view of the following developments, a discrete-time implementation of the residual (10) with Tustin rule will be used. At time $t_{k}=k T$, with $T$ being the sampling time, the residual $r_{\tau_{e}}(k)=r_{\tau_{e}}\left(t_{k}\right)$ is computed as:

$$
\begin{aligned}
I_{\tau}(k) & =I_{\tau}(k-1)+\frac{\tau(k)+\tau(k-1)}{2} T \\
r(k) & =K_{\tau_{e}}\left(B \dot{\theta}(k)+D_{\theta} \theta(k)-I_{\tau}(k)\right) \\
r_{\tau_{e}}(k) & =\frac{2-T K_{\tau_{e}}}{2+T K_{\tau_{e}}} r_{\tau_{e}}(k-1)+\frac{2(r(k)-r(k-1))}{2+T K_{\tau_{e}}} .
\end{aligned}
$$

For a VSA device having two flexible transmissions, we can design an independent residual (10) for each transmission from the motor side, and then use eq. (8) to obtain the total flexibility torque. The same scheme applies in a decentralized way for a robot with multiple joints driven by VSA devices.

\section{RECURSIVE LEAST SQUARES FOR STIFFNESS ESTIMATION}

In principle, the transmission stiffness could be obtained from the time derivative of the measured flexibility torque

$$
\dot{\tau}_{e}(\phi)=\frac{\partial \tau_{e}}{\partial \phi} \dot{\phi}=\sigma(\phi) \dot{\phi}
$$

and by processing (13) so as to extrapolate the stiffness, as proposed by Grioli and Bicchi (2010). Similarly, in the absence of a torque sensor, one could use the residual $r_{\tau_{e}}$ in (10) as an estimate replacing the flexibility torque $\tau_{e}$ in (13). However, this approach appears inadequate due to measurement errors and noise introduced by the numerical differentiation.

We propose instead to approximate the flexibility torque with a nonlinear function of the transmission deformation $\phi$, parametrized by a $n$-vector of constant and still unknown parameters $\boldsymbol{\alpha}=\left(\alpha_{1} \ldots \alpha_{n}\right)^{T}$, i.e.,

$$
\tau_{e}(\phi) \simeq f(\phi, \boldsymbol{\alpha}) \text {. }
$$

If the vector $\boldsymbol{\alpha}$ were known, the stiffness could be analytically computed from (2) and (14) as

$$
\sigma(\phi)=\frac{\partial f(\phi, \boldsymbol{\alpha})}{\partial \phi} .
$$

The functional estimation of the transmission stiffness is then converted into a parametric estimation of vector $\boldsymbol{\alpha}$. Similarly, one can obtain analytically also successive derivatives of the stiffness $\sigma$ w.r.t. $\phi$, which may be needed for control purposes (see Sect. 6).

For the structure of $f$ in eq. (14), we use a linear parameterization in $\boldsymbol{\alpha}$, i.e.,

$$
f(\phi, \boldsymbol{\alpha})=\sum_{h=1}^{n} f_{h}(\phi) \alpha_{h}=\boldsymbol{F}^{T}(\phi) \boldsymbol{\alpha},
$$

where the row vector $\boldsymbol{F}^{T}(\phi)=\partial f / \partial \boldsymbol{\alpha}$ is the associated Jacobian. The set of basis functions $f_{h}, h=1, \ldots, n$, and its number $n$ have to be chosen appropriately: polynomials 
of increasing order are a general and convenient choice. In particular, taking into account the physical property (1) of the flexibility torque, we select only the first $n$ odd powers of $\phi$ up to the order $2 n-1$ :

$$
f_{h}(\phi)=\phi^{2 h-1}, \quad h=1, \ldots, n .
$$

To obtain an estimate $\widehat{\boldsymbol{\alpha}}$ of the parameter vector $\boldsymbol{\alpha}$, we adopt a least squares method in discrete time. At time $t_{k}=k T$, the deformation $\phi(k)=\phi\left(t_{k}\right)$ and the residual $r_{\tau_{e}}(k)=r_{\tau_{e}}\left(t_{k}\right)$ are collected as data points. The parameters estimate $\widehat{\boldsymbol{\alpha}}$ is chosen so as to minimize the cost function $E$ given by the sum of the squared differences between the observed residuals and the estimated transmission flexibility torque, i.e.,

$$
E=\frac{1}{2} \sum_{k=1}^{p}\left(r_{\tau_{e}}(k)-f(\phi(k), \widehat{\boldsymbol{\alpha}})\right)^{2},
$$

where $p>n$ is a sufficient number of collected data points. The solution of the estimation problem in batch form is

$$
\widehat{\boldsymbol{\alpha}}=\left(\sum_{k=1}^{p} \boldsymbol{F}(k) \boldsymbol{F}^{T}(k)\right)^{-1}\left(\sum_{k=1}^{p} \boldsymbol{F}(k) r_{\tau_{e}}(k)\right),
$$

where the Jacobian $\boldsymbol{F}^{T}(k)$ is

$$
\boldsymbol{F}^{T}(k)=\left(\begin{array}{llll}
\phi(k) & \phi^{3}(k) \ldots & \phi^{2 n-1}(k)
\end{array}\right) .
$$

Since on-line parameter estimation is more suitable for control purposes, the estimate (19) should be updated recursively at each sampling time. The standard Recursive Least Squares (RLS) algorithm is

$$
\widehat{\boldsymbol{\alpha}}(k)=\widehat{\boldsymbol{\alpha}}(k-1)+\Delta \widehat{\boldsymbol{\alpha}}(k),
$$

with

$$
\Delta \widehat{\boldsymbol{\alpha}}(k)=\boldsymbol{L}(k)\left(r_{\tau_{e}}(k)-\boldsymbol{F}^{T}(k) \widehat{\boldsymbol{\alpha}}(k-1)\right)
$$

where

$$
\boldsymbol{L}(k)=\frac{\boldsymbol{P}(k-1) \boldsymbol{F}(k)}{1+\boldsymbol{F}^{T}(k) \boldsymbol{P}(k-1) \boldsymbol{F}(k)}
$$

and

$$
\boldsymbol{P}(k)=\left(\boldsymbol{I}-\boldsymbol{L}(k) \boldsymbol{F}^{T}(k)\right) \boldsymbol{P}(k-1),
$$

being $\boldsymbol{P}(k)$ the $n \times n$ covariance matrix and $\boldsymbol{I}$ the identity matrix. As opposed to (19), the recursive algorithm suffers less from possible ill-conditioning of $\boldsymbol{F}$. The algorithm is initialized with an a priori estimate $\widehat{\boldsymbol{\alpha}}(0)$ and a positive definite (usually, diagonal) choice $\boldsymbol{P}(0)$ for the covariance matrix. This matrix is typically initialized with large values, considering no a priori knowledge about the true parameter vector $\boldsymbol{\alpha}$. For a convergence analysis of the RLS algorithm, see, e.g., Johnson (1988).

The degree $n$ of the polynomial $f(\phi(k), \boldsymbol{\alpha})$ in (16) should be large enough to capture the nonlinearities of the transmission flexibility torque, especially if large deformations occur. Otherwise, even when the estimate converges and the covariance matrix $\boldsymbol{P}$ becomes small, the RLS estimate will no longer track efficiently the residual data (see also the example in Sect. 5). The appropriate degree of the polynomial approximation can be tested using a number of indicators, the simplest of which is $E$ itself in (18).

Summarizing, for any parametrized model (16) used to approximate the flexibility torque, the current estimate $\widehat{\boldsymbol{\alpha}}(k)$ obtained with the RLS algorithm provides a stiffness estimate given by

$$
\widehat{\sigma}(k)=\frac{\partial f(\phi, \widehat{\boldsymbol{\alpha}}(k))}{\partial \phi}=\sum_{h=1}^{n} \frac{\partial f_{h}(\phi)}{\partial \phi} \widehat{\alpha}_{h}(k) .
$$

Indeed, this stiffness estimation procedure can be applied (simultaneously) to both transmissions of the VSA system, yielding $\widehat{\sigma}_{1}(k)$ and $\widehat{\sigma}_{2}(k)$ and thus $\widehat{\sigma}_{t}(k)=\widehat{\sigma}_{1}(k)+\widehat{\sigma}_{2}(k)$ for the total device stiffness, according to (9).

\subsection{Residual Error Recovery (RER)}

A simple improvement of the residual-based RLS estimation of the flexibility torque, and thus of the transmission stiffness, can be obtained as follows. From eq. (11), the residual error can be expressed as

$$
\epsilon_{r}=\tau_{e}(\phi)-r_{\tau_{e}}=\frac{\dot{r}_{\tau_{e}}}{K_{\tau_{e}}}
$$

Therefore, a first possibility is to approximate the residual error $\epsilon_{r}$ with backward differences in discrete time, namely using

$$
\epsilon_{r}(k)=\frac{r_{\tau_{e}}(k)-r_{\tau_{e}}(k-1)}{T K_{\tau_{e}}} .
$$

A second possibility is based on the approximate relation

$$
\dot{r}_{\tau_{e}} \approx \dot{\tau}_{e}(\phi) \text {. }
$$

Using (13), with the current estimate $\widehat{\sigma}(k)$ given by $(25)$ and a measure of $\dot{\phi}(k)=\dot{q}(k)-\dot{\theta}(k)$, an estimate of the residual error $\epsilon_{r}$ in (26) is provided at each step $k$ by

$$
\epsilon_{r}(k)=\frac{\widehat{\sigma}(k) \dot{\phi}(k)}{K_{\tau_{e}}} .
$$

The choice between (27) and (29) depends on the kind of sensors used and on the measurement noise. In any event, we can use $\epsilon_{r}(k)$ to compensate for the time lag introduced by the residual, replacing (22) with

$$
\Delta \widehat{\boldsymbol{\alpha}}(k)=\boldsymbol{L}(k)\left(r_{\tau_{e}}(k)+\epsilon_{r}(k)-\boldsymbol{F}^{T}(k) \widehat{\boldsymbol{\alpha}}(k-1)\right) .
$$

\section{ESTIMATION RESULTS}

We illustrate the performance of the proposed residualbased RLS estimation of stiffness on the VSA-II device developed by Schiavi et al. (2008). In this antagonistic VSA device, the nonlinear characteristics of each of the two flexible transmissions is obtained thanks to a pair of 4-bar linkages with linear springs. The flexibility torque of each transmission can be modeled as

$$
\tau_{e, i}\left(\phi_{i}\right)=2 k_{i} \beta\left(\phi_{i}\right) \frac{\partial \beta\left(\phi_{i}\right)}{\partial \phi_{i}}, \quad i=1,2,
$$

where $k_{i}>0$ is the constant stiffness of the spring in the $i$-th transmission, and

$$
\beta\left(\phi_{i}\right)=\arcsin \left(C_{i} \sin \left(\frac{\phi_{i}}{2}\right)\right)-\frac{\phi_{i}}{2}, \quad i=1,2,
$$

being $C_{i}>1$ a geometric non-dimensional parameter of the linkage. The total flexibility torque $\tau_{e, t}$ acting on the link dynamics (5) is given by eq. (8). Note that in the model of $\tau_{e, i}$ the two uncertain parameters $k_{i}$ and $C_{i}$ appear, respectively, in a linear and nonlinear way.

The nominal dynamic parameters for the simulations of this robotic system are those reported by Schiavi et al. (2008). In particular, the four parameters that characterize the flexible transmissions are $C_{i}=1.75$ and $k_{i}=$ 


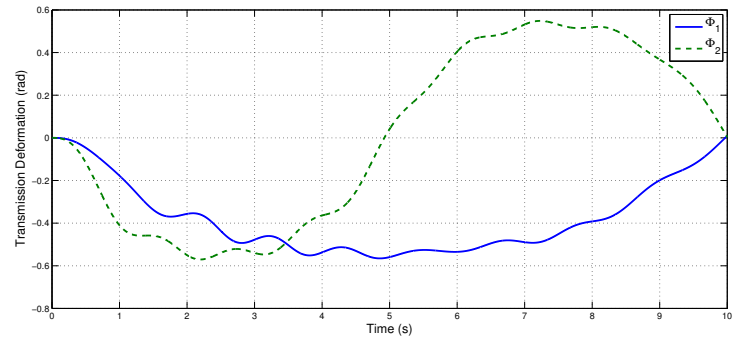

Fig. 3. Transmission deformations $\phi_{1}, \phi_{2}$ for the VSA-II device under the sinusoidal torque inputs (33)

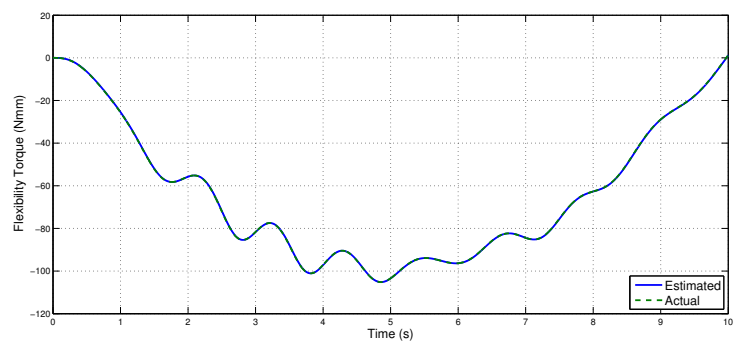

(a)

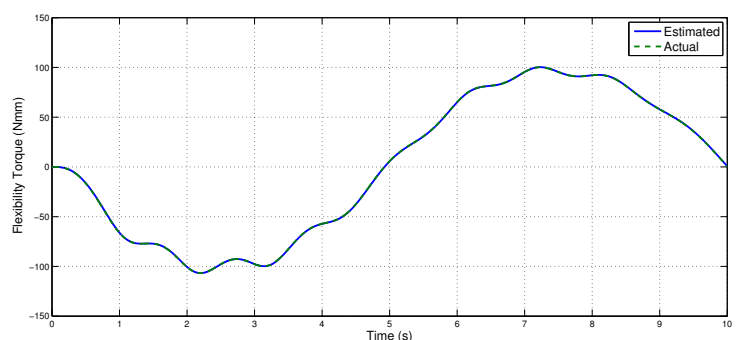

(b)

Fig. 4. Comparison of residual $r_{\tau_{e}}$ in (12) and nominal flexibility torque $\tau_{e}$ for the first (a) and second (b) transmission

$500[\mathrm{~N} \cdot \mathrm{mm} / \mathrm{rad}]$, for $i=1,2$. Applying for $10 \mathrm{sec}$ the openloop torques

$$
\tau_{1}=10 \cdot \sin 0.1 \pi \quad \tau_{2}=10 \cdot \sin 0.2 \pi \quad[\mathrm{N} \cdot \mathrm{mm}],
$$

starting from an undeformed equilibrium configuration with the link pointing downward (under gravity), we obtained the transmission deformations $\phi_{1}$ and $\phi_{2}$ shown in Fig. 3. Figure 4 compares the associated residuals computed by (12), using $K_{\tau_{e}}=300$ and a sampling time $T=0.1 \mathrm{msec}$, with the evolution of the nominal flexibility torques of the two transmissions. No differences can be appreciated in practice.

For stiffness estimation, the residual-based RLS algorithm (21-24) has been applied with $n=4$ polynomial terms in (16-17), an initial parameter estimate $\widehat{\boldsymbol{\alpha}}(0)=\mathbf{0}$ and an initial $4 \times 4$ covariance matrix $\boldsymbol{P}(0)=10^{6} \boldsymbol{I}$. For each of the two VSA-II transmissions, Figures 5 and 6 show the time evolution of the estimated stiffness in comparison with the nominal one and a few snapshots (at $t=0.5,1$, and $10 \mathrm{sec}$ ) of how the approximating function progresses toward the nominal stiffness characteristic. Convergence to the actual current value of stiffness occurs within one third of the total motion time. On the other hand, the

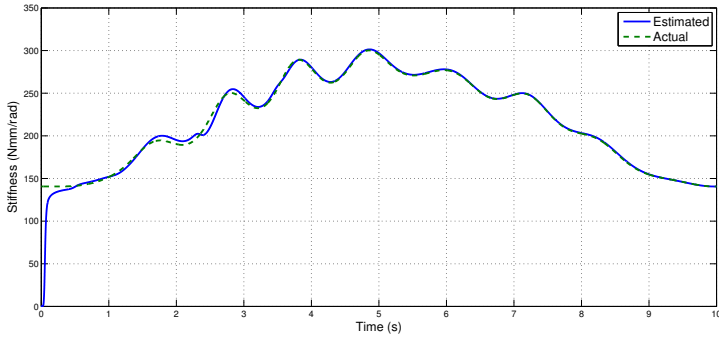

(a)

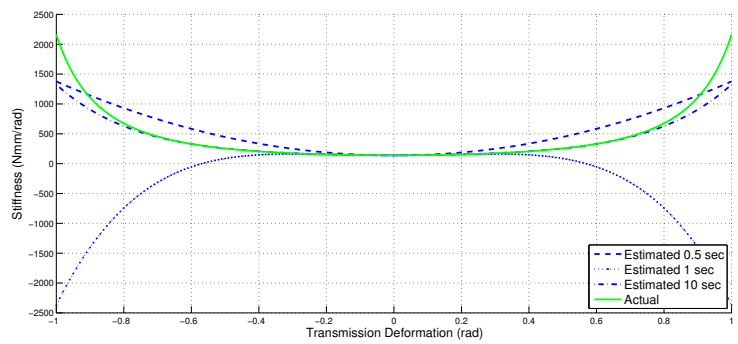

(b)

Fig. 5. Comparison of estimated and nominal stiffness (a) and the approximating stiffness function at selected instants (b) for the first transmission of the VSA-II

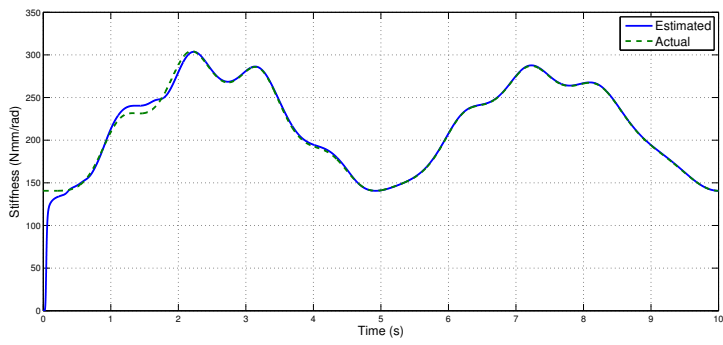

(a)

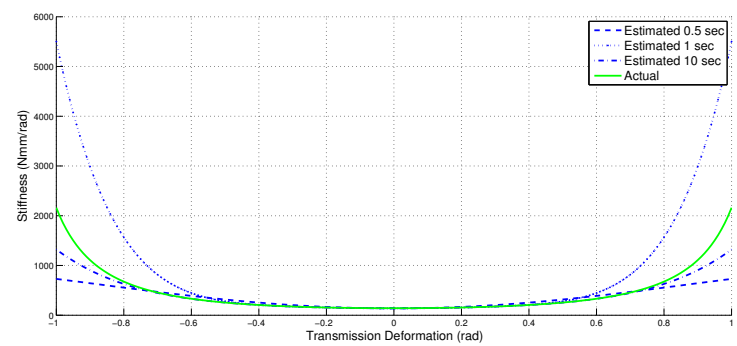

(b)

Fig. 6. Same plots as in Fig. 5 for the second transmission

curve fitting of the complete characteristics is poor for deformations that are larger in module than $0.6 \mathrm{rad}$. This should not be unexpected since such large deformations (see Fig. 3) do not occur during the specific motion obtained under the torque commands (33).

Next, we have evaluated the effects of the residual error recovery scheme of Sect. 4.1 in the RLS stiffness estimator. Figures 7 and 8 show the benefit obtained when using (30). The time needed to converge to the the nominal stiffness is largely reduced, while spurious transient phenomena 


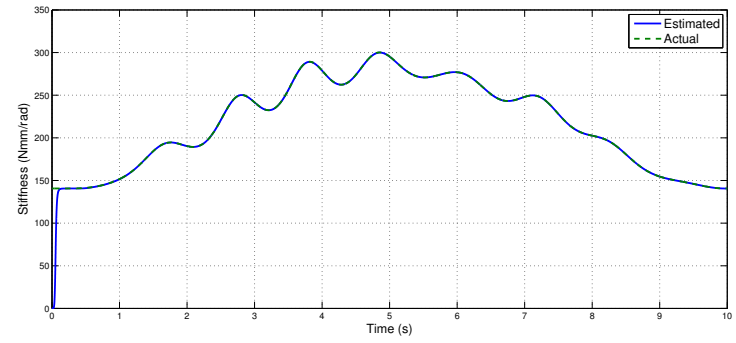

(a)

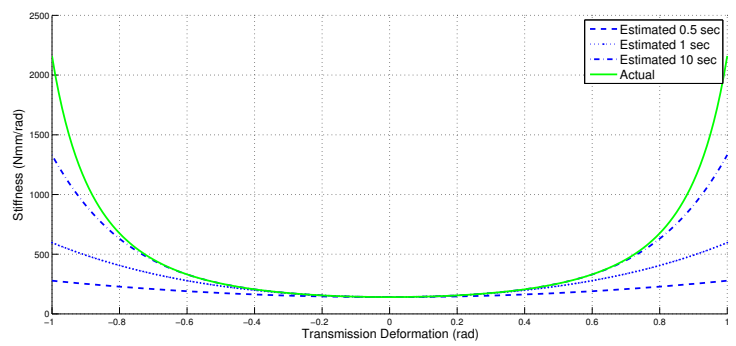

(b)

Fig. 7. Comparison of estimated and nominal stiffness (a) and the approximating stiffness function at selected instants (b) for the first transmission of the VSA-II, when using the residual error recovery

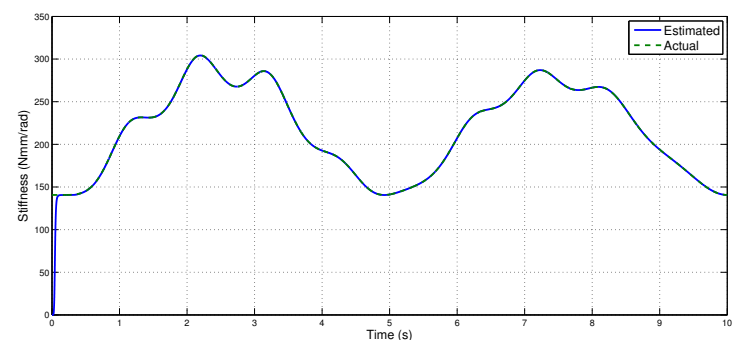

(a)

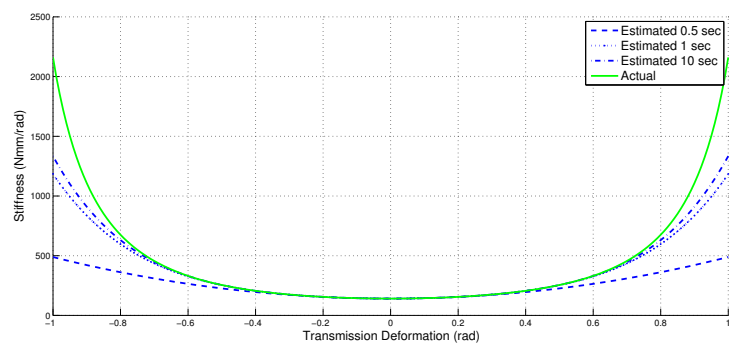

(b)

Fig. 8. Same plots as in Fig. 7 for the second transmission

on the approximating function are eliminated - compare, e.g., the situation at $t=1 \mathrm{sec}$ in Figs. 5(b) and 7(b).

Finally, to illustrate the problems of an under-parametrized model, we report in Fig. 9 the results on stiffness estimation when the number of terms in the polynomial (16-17) approximating the flexibility torque is reduced to $n=2$. A permanent and recurrent error is clearly left.

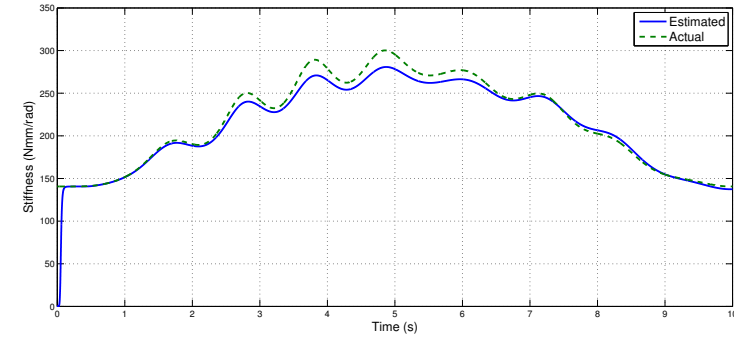

(a)

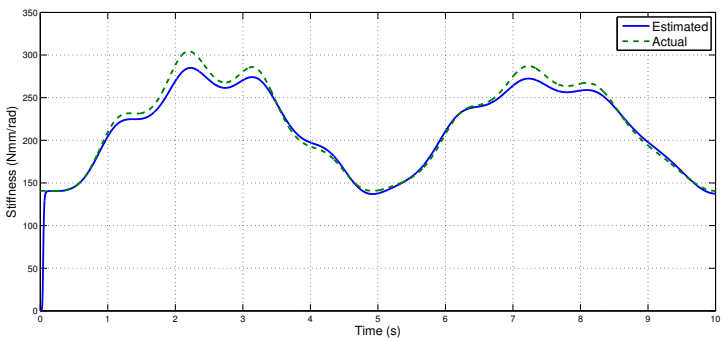

(b)

Fig. 9. Stiffness estimation for the VSA-II device when using $n=2$ terms in the polynomial approximation (16-17); first (a) and second (b) transmission

\section{FEEDBACK LINEARIZATION CONTROL}

In this section, we combine the proposed on-line stiffness estimator with the feedback linearization (FBL) controller for the VSA-II robotic system introduced by De Luca et al. (2009). For the details on the feedback linearization design, the reader is referred to the original publication. The linearizing coordinates for system (5-6) are the link position $q$ together with its first three time derivatives, and the device stiffness $\sigma_{t}$ with its first derivative. The state $\boldsymbol{x}=\left(q, \dot{q}, \theta_{1}, \dot{\theta}_{1}, \theta_{2}, \dot{\theta}_{2}\right)$ is thus diffeomorphic to the transformed state $\boldsymbol{z}=\left(q, \dot{q}, \ddot{q}, q^{[3]}, \sigma_{t}, \dot{\sigma}_{t}\right)$. Therefore, the dynamics of the VSA-II system can be rewritten as

$$
\left(\begin{array}{c}
q^{[4]} \\
\ddot{\sigma}_{t}
\end{array}\right)=\mathcal{A}(\boldsymbol{x})\left(\begin{array}{c}
\tau_{1} \\
\tau_{2}
\end{array}\right)+\boldsymbol{b}(\boldsymbol{x}),
$$

where $\mathcal{A}(\boldsymbol{x})$ is the so called decoupling matrix

$$
\mathcal{A}(\boldsymbol{x})=\boldsymbol{\Gamma}\left(\begin{array}{cc}
\sigma_{1} & \sigma_{2} \\
\frac{\partial \sigma_{1}}{\partial \phi_{1}} & \frac{\partial \sigma_{2}}{\partial \phi_{2}}
\end{array}\right),
$$

being $\Gamma$ a constant, diagonal, and invertible matrix, and $\boldsymbol{b}(\boldsymbol{x})$ a computable function of $\boldsymbol{x}$. The complete expressions of $\boldsymbol{\Gamma}$ and $\boldsymbol{b}(\boldsymbol{x})$ are given in Appendix A. It can be shown that the decoupling matrix is always invertible provided that $\phi_{1} \neq \phi_{2}$, a condition that can be safely avoided by a suitable pre-charging of the system.

The dynamic equations (34) can be exactly linearized (and input-output decoupled) using the nonlinear state feedback law

$$
\left(\begin{array}{c}
\tau_{1} \\
\tau_{2}
\end{array}\right)=\mathcal{A}^{-1}(\boldsymbol{x})\left(\left(\begin{array}{l}
v_{1} \\
v_{2}
\end{array}\right)-\boldsymbol{b}(\boldsymbol{x})\right),
$$

where $v_{1}$ and $v_{2}$ are the new control inputs. These can be designed for stable trajectory tracking purposes on the linear and decoupled side of the problem. In fact, the closed-loop system given by (34) and (36) is made 
by two independent chains of input-output integrators (four integrators between $v_{1}$ and the link position $q$ and two between $v_{2}$ and the device stiffness $\sigma_{t}$ ). The tracking errors with respect to smooth reference trajectories $q_{d}(t)$ for the link position and $\sigma_{t, d}(t)$ for the device stiffness is exponentially stabilized by a $\mathrm{PD}^{3}$ error feedback law for $v_{1}$ (with suitable positive gains characterizing a Hurwitz polynomial) and a PD error feedback law for $v_{2}$ (with positive gains), plus the feedforward terms, respectively $q_{d}^{[4]}(t)$ and $\ddot{\sigma}_{t, d}(t)$.

Assume now that all dynamic parameters in eqs. (5-6) are known, except for those related to the transmission flexibility. The unknown components required for implementing the feedback linearization law (36) are the flexibility torques $\tau_{e_{1}}$ and $\tau_{e_{2}}$ and the transmission stiffnesses $\sigma_{1}$ and $\sigma_{2}$, together with their first and second derivatives w.r.t. the deformations $\phi_{1}$ and $\phi_{2}$. More explicitly, we need $\frac{\partial \sigma_{1}}{\partial \phi_{1}}$, $\frac{\partial \sigma_{2}}{\partial \phi_{2}}, \frac{\partial^{2} \sigma_{1}}{\partial \phi_{1}^{2}}$, and $\frac{\partial^{2} \sigma_{2}}{\partial \phi_{2}^{2}}$. The last two quantities are used in the evaluation of $\boldsymbol{b}(\boldsymbol{x})$, see again Appendix A.

Using the proposed estimator, for each transmission of the VSA-II we can evaluate (dropping the index $i=1,2$ )

$$
\begin{aligned}
\widehat{\tau}_{e}(\phi) & =f(\phi, \widehat{\boldsymbol{\alpha}})=\sum_{h=1}^{n} \phi^{2 h-1} \widehat{\alpha}_{h} \\
\widehat{\sigma}(\phi) & =\sum_{h=1}^{n}(2 h-1) \phi^{2 h-2} \widehat{\alpha}_{h} \\
\frac{\partial \widehat{\sigma}(\phi)}{\partial \phi} & =\sum_{h=2}^{n}\left(4 h^{2}-6 h+2\right) \phi^{2 h-3} \widehat{\alpha}_{h} \\
\frac{\partial^{2} \widehat{\sigma}(\phi)}{\partial \phi^{2}} & =\sum_{h=2}^{n}\left(8 h^{3}-24 h^{2}+22 h-6\right) \phi^{2 h-4} \widehat{\alpha}_{h},
\end{aligned}
$$

and insert these in place of the unknown terms. The integration of the proposed estimator within the FBL controller leads to the scheme shown in Fig. 1.

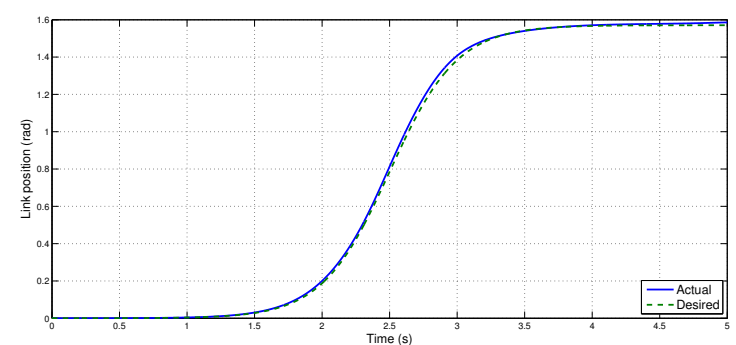

(a)

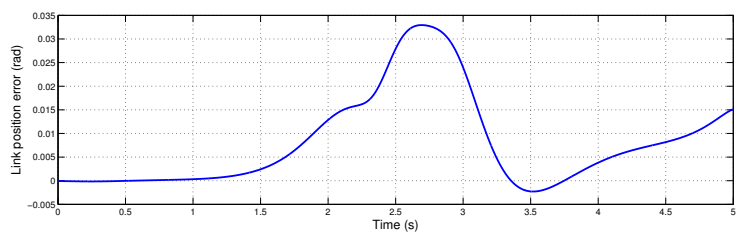

(b)

Fig. 10. Reference and actual link position with FBL control using stiffness estimation (a) and link trajectory tracking error (b)

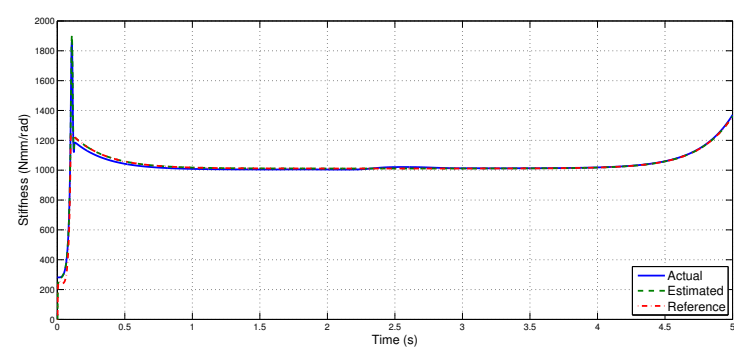

(a)

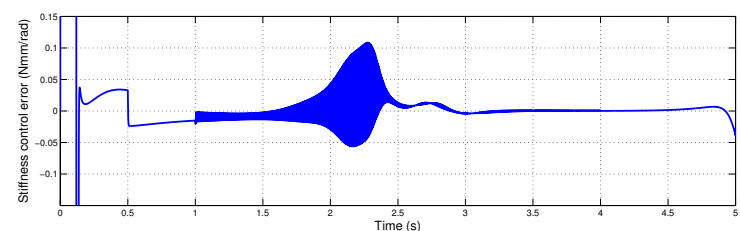

(b)

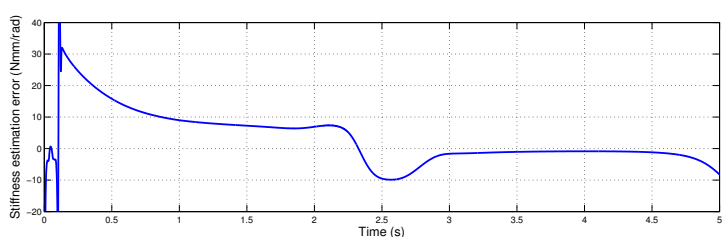

(c)

Fig. 11. Reference, estimated, and actual stiffness trajectory obtained with FBL control using stiffness estimation (a), reference-estimated stiffness control error (b), and stiffness estimation error (c)

In the performed simulations, we have used the same reference trajectories, linear control gains, and initial error conditions used by De Luca et al. (2009). For precharging the system, in order to avoid a singularity of the decoupling matrix, the commands $\tau_{1}=1.2[\mathrm{~N} \cdot \mathrm{mm}]$ and $\tau_{2}=-1.2[\mathrm{~N} \cdot \mathrm{mm}]$ have been applied for $0.1 \mathrm{sec}$, before turning the FBL controller on. Figure 10 shows the pointto-point motion of the link and its reference trajectory, as well as the small obtained position error. The reference stiffness trajectory, the controlled evolution of the stiffness based on its estimation, and the actual (nominal) stiffness evolution are given in Fig. 11, together with the errors between the reference and estimated stiffness output and between the actual and estimated stiffness. When the FBL control is activated, the error between the reference and the estimated stiffness output is extremely small and remains practically negligible thanks to the control action (less than $0.01 \%$ in Fig. 11(b)). Also the stiffness estimation error (actual vs. estimated) is relatively small, with a maximum of about $2 \%$ (Fig. 11(c)).

We conclude this section with a remark on the closedloop stability of the complete estimation-control scheme. While a formal proof cannot be provided at this stage, the exponential stability of the flexibility torque estimator, the fast convergence of the RLS stiffness estimator with a sufficiently rich parameterization and under persistency of excitation, as well as the exponential tracking properties of the feedback linearization control in nominal conditions, are fundamental features that allow to predict that practical stability is guaranteed. 


\section{CONCLUSIONS}

A novel on-line stiffness estimation method for robots with variable stiffness actuation in agonistic-antagonistic configuration has been proposed, and its output has been combined with an advanced motion/stiffness controller based on feedback linearization and input-output decoupling. Since the stiffness of each transmission is estimated independently and locally at each motor side, the estimator requires no information on the link dynamics and limited sensing, in particular no joint torque sensor. The integration of the stiffness estimator with the feedback linearizing controller has been presented for a single-link device (also with non-symmetric dynamic and flexibility properties), but can be extended to the case of multi-link robots using VSA in a straightforward way.

After the satisfactory results obtained in simulations, we are planning an experimental verification on the VSA-II system, in collaboration with the University of Pisa. Moreover, we are currently extending the estimation approach also to other VSA configurations, e.g., using harmonic drives with variable stiffness (Wolf and Hirzinger (2008); Catalano et al. (2010)). In Flacco et al. (2011), we present preliminary experiments on the stiffness estimation for the IIT AwAS, a VSA device in serial configuration where a primary motor controls link motion and a secondary motor is used to adjust stiffness.

\section{REFERENCES}

Bicchi, A. and Tonietti, G. (2004). Fast and soft arm tactics: Dealing with the safety-performance trade-off in robot arms design and control. IEEE Robotics and Automation Mag., 11(2), 22-33.

Catalano, M., Schiavi, R., and Bicchi, A. (2010). Design of variable stiffness actuators mechanisms based on enumeration and analysis of performance. In Proc. IEEE Int. Conf. on Robotics and Automation, 3285-3291.

Coutinho, F. and Cortesao, R. (2010). System stiffness estimation with the candidate observers algorithm. In Proc. 18th Mediterranean Conf. on Control and Automation, 796-801.

De Luca, A. and Book, W. (2008). Robots with flexible elements. In B. Siciliano and O. Khatib (eds.), Springer Handbook of Robotics, 287-319. Springer.

De Luca, A., Flacco, F., Bicchi, A., and Schiavi, R. (2009). Nonlinear decoupled motion-stiffness control and collision detection/reaction for the VSA-II variable stiffness device. In Proc. IEEE/RSJ Int. Conf. on Intelligent Robots and Systems, 5487-5494.

De Luca, A. and Mattone, R. (2003). Actuator failure detection and isolation using generalized momenta. In Proc. IEEE Int. Conf. on Robotics and Automation, 634-639.

De Santis, A., Siciliano, B., De Luca, A., and Bicchi, A. (2008). An atlas of physical human-robot interaction. Mechanism and Machine Theory, 43(3), 253-270.

Diolaiti, N., Melchiorri, C., and Stramigioli, S. (2005). Contact impedance estimation for robotic systems. IEEE Trans. on Robotics, 21(5), 925-935.

Flacco, F. and De Luca, A. (2011). Residual-based stiffness estimation in robots with flexible transmissions. To be presented at IEEE Int. Conf. on Robotics and Automation, Shanghai, PRC (May 2011).
Flacco, F., De Luca, A., Sardellitti, I., and Tsagarakis, N. (2011). Robust estimation of variable stiffness in flexible joints. Submitted to IEEE/RSJ Int. Conf. on Intelligent Robots and Systems, San Francisco, CA (Sep. 2011).

Grioli, G. and Bicchi, A. (2010). A non-invasive realtime method for measuring variable stiffness. In Proc. Robotics Science and Systems (RSS 2010), Zaragoza, E.

Haddadin, S., Albu-Schäffer, A., De Luca, A., and Hirzinger, G. (2008). Collision detection and reaction: A contribution to safe physical human-robot interaction. In Proc. IEEE/RSJ Int. Conf. on Intelligent Robots and Systems, 3356-3363.

Johnson, C. (1988). Lectures on Adaptive Parameter Estimation. Prentice Hall.

Ludvig, D. and Kearney, R.E. (2009). Estimation of joint stiffness with a compliant load. In Proc. 31st IEEE Int. Conf. on EMBS, 2967-2970.

Schiavi, R., Grioli, G., Sen, S., and Bicchi, A. (2008). VSAII: A novel prototype of variable stiffness actuator for safe and performing robots interacting with humans. In Proc. IEEE Int. Conf. on Robotics and Automation, $2171-2176$

Tonietti, G., Schiavi, R., and Bicchi, A. (2005). Design and control of a variable stiffness actuator for safe and fast physical human/robot interaction. In Proc. IEEE Int. Conf. on Robotics and Automation, 528-533.

Verscheure, D., Scharf, I., Bruyninckx, H., Swevers, J., and De Schutter, J. (2009). Identification of contact dynamics parameters for stiff robotic payloads. IEEE Trans. on Robotics, 25(2), 240-252.

Wolf, S. and Hirzinger, G. (2008). A new variable stiffness design: Matching requirements of the next robot generation. In Proc. IEEE Int. Conf. on Robotics and Automation, 1741-1746.

\section{Appendix A. FEEDBACK LINEARIZATION TERMS}

The remaining terms of the feedback linearization law (36) of the VSA-II system are given. We have

$$
\boldsymbol{\Gamma}=\left(\begin{array}{cc}
\frac{1}{B M} & 0 \\
0 & -\frac{1}{B}
\end{array}\right),
$$

while, for $\boldsymbol{b}(\boldsymbol{x})=\left(b_{q}(\boldsymbol{x}) b_{\sigma}(\boldsymbol{x})\right)^{T}$,

$$
\begin{aligned}
b_{q}(\boldsymbol{x})= & -\frac{1}{M}\left(\frac{\sigma_{1}}{B}\left(D_{\theta} \dot{\theta}_{1}-\tau_{e_{1}}\right)+\frac{\sigma_{2}}{B}\left(D_{\theta} \dot{\theta}_{2}-\tau_{e_{2}}\right)\right. \\
& \left.+\left(\sigma_{1}+\sigma_{2}+D_{q}\right) \ddot{q}+\frac{\partial \sigma_{1}}{\partial \phi_{1}} \dot{\phi}_{1}^{2}+\frac{\partial \sigma_{2}}{\partial \phi_{2}} \dot{\phi}_{2}^{2}+\ddot{g}(q)\right)
\end{aligned}
$$

and

$$
\begin{aligned}
b_{\sigma}(\boldsymbol{x})= & -\frac{1}{B}\left(\frac{\partial \sigma_{1}}{\partial \phi_{1}}\left(\tau_{e_{1}}-D_{\theta} \dot{\theta}_{1}\right)+\frac{\partial \sigma_{2}}{\partial \phi_{2}}\left(\tau_{e_{2}}-D_{\theta} \dot{\theta}_{2}\right)\right) \\
& +\left(\frac{\partial \sigma_{1}}{\partial \phi_{1}}+\frac{\partial \sigma_{2}}{\partial \phi_{2}}\right) \ddot{q}+\frac{\partial^{2} \sigma_{1}}{\partial \phi_{1}^{2}} \dot{\phi}_{1}^{2}+\frac{\partial^{2} \sigma_{2}}{\partial \phi_{2}^{2}} \dot{\phi}_{2}^{2} .
\end{aligned}
$$

The link acceleration $\ddot{q}$, which appears explicitly in (A.2) and (A.3) and through $\ddot{g}(q)$ in (A.2), is evaluated from eq. (5) and will thus depend also on $\tau_{e, t}(\phi)$. 